Research article

\title{
The ubiquitin-like molecule interferon-stimulated gene 15 (ISG15) is a potential prognostic marker in human breast cancer
}

\author{
Nuran Bektas ${ }^{1}$, Erik Noetzel ${ }^{1}$, Jürgen Veeck¹, Michael F Press ${ }^{2}$, Glen Kristiansen³ ${ }^{3}$ Amjad Naami ${ }^{1}$, \\ Arndt Hartmann ${ }^{4}$, Arno Dimmler ${ }^{4}$, Matthias W Beckmann ${ }^{5}$, Ruth Knüchel ${ }^{1}$, Peter A Fasching ${ }^{5}$ and \\ Edgar Dahl 1
}

\author{
1'Department of Pathology, University Hospital of the RWTH Aachen, Pauwelsstrasse 30, 52074 Aachen, Germany \\ 2Department of Pathology, Keck School of Medicine, University of Southern California/Norris Comprehensive Cancer Center, 1441 Eastlake Avenue, \\ Los Angeles, CA 90033, USA \\ ${ }^{3}$ Department of Pathology, University Hospital Zürich, Schmelzbergstrasse 12, 8091 Zürich, Switzerland \\ ${ }^{4}$ Department of Pathology, University Hospital Erlangen, Krankenhausstrasse 12, 91054 Erlangen, Germany \\ 5Department of Gynaecology, University Hospital Erlangen, Universitätsstrasse 21-23, 91054 Erlangen, Germany \\ Corresponding author: Edgar Dahl, edahl@ukaachen.de
}

Received: 21 Mar 2008 Revisions requested: 30 Apr 2008 Revisions received: 20 May 2008 Accepted: 15 Jul 2008 Published: 15 Jul 2008

Breast Cancer Research 2008, 10:R58 (doi:10.1186/bcr2117)

This article is online at: http://breast-cancer-research.com/content/10/4/R58

(c) 2008 Bektas et al.; licensee BioMed Central Ltd.

This is an open access article distributed under the terms of the Creative Commons Attribution License (http://creativecommons.org/licenses/by/2.0), which permits unrestricted use, distribution, and reproduction in any medium, provided the original work is properly cited.

\begin{abstract}
Introduction ISG15 is an ubiquitin-like molecule that is strongly upregulated by type I interferons as a primary response to diverse microbial and cellular stress stimuli. However, alterations in the ISG15 signalling pathway have also been found in several human tumour entities. To the best of our knowledge, in the current study we present for the first time a systematic characterisation of ISG15 expression in human breast cancer and normal breast tissue both at the mRNA and protein level.
\end{abstract}

Method Using semiquantitative real-time PCR, cDNA dot-blot hybridisation and immunohistochemistry, we systematically analysed ISG15 expression in invasive breast carcinomas ( $n=$ 910) and normal breast tissues $(n=135)$. ISG15 protein expression was analysed in two independent cohorts on tissue microarrays; in an initial evaluation set of 179 breast carcinomas and 51 normal breast tissues; and in a second large validation set of 646 breast carcinomas and 10 normal breast tissues. In addition, a collection of benign and malignant mammary cell lines $(n=9)$ were investigated for ISG15 expression.

Results ISG15 was overexpressed in breast carcinoma cells compared with normal breast tissue, both at the RNA and protein level. Recurrence-free $(p=0.030)$, event-free $(p=$ $0.001)$ and overall $(p=0.001)$ survival analyses showed a significant correlation between ISG15 overexpression and unfavourable prognosis.

Conclusion Therefore, ISG15 may represent a novel breast tumour marker with prognostic significance and may be helpful in selecting patients for and predicting response to the treatment of human breast cancer.

\section{Introduction}

The interferon-stimulated gene $15 \mathrm{kDa}$ (ISG15) is an interferon-inducible ubiquitin-like protein. In addition to being stimulated by type I interferon, expression of ISG15 is greatly induced by viral or bacterial infection through the Janus kinase/signal transducer and activator of transcription (Jakl STAT) signalling pathway [1-3]. After induction, ISG15 is secreted by monocytes, B- and T-lymphocytes and fibroblasts.
The ISG15 protein is made up of tandem ubiquitin homology domains [4]. Within cells, ISG15 is covalently conjugated with cellular proteins in an enzymatic pathway (so called ISGylation) comprised of the activating E1, conjugating E2 and ligating E3 enzymes, which are similar to those used by ubiquitin (ubiquitylation). The conjugation of ISG15 with protein substrates provides a tag that either marks the labelled protein for degradation [5] or modulates its function [6]. Importantly,

$\overline{\mathrm{BBCC}}=$ Bavarian Breast Cancer Cases and Controls; EFS = Event-free survival; ER = Oestrogen receptor; H\&E = Haematoxylin and eosin; IRS = Immunoreactivity Score; ISG15 = Interferon-stimulated gene $15 \mathrm{kDa}$; OS = Overall survival; PCR = polymerase chain reaction; RFS = Recurrencefree survival; TMA = Tissue microarray. 
protein ISGylation is a reversible process, and removal of ISG15 molecules is mediated by de-ISGylating enzymes [7].

Multiple target proteins of ISG15 have recently been identified, some of which have been implicated in the regulation of immune responses. However, the biological consequences of modification by ISG15 need to be determined further [8]. Interestingly, protein ubiquitylation has been shown to be of fundamental importance in the regulation of both the innate and adaptive immune systems, with roles in the control of immune tolerance, the differentiation of T-cells and the intracellular signal transduction induced by antigen, cytokines or tolllike receptor ligands [9].

So far, little is known about the function of ISG15 in carcinogenesis. ISG15 is a post-translational protein modifier and also a secreted cytokine. Each of these roles has been implicated in carcinogenesis, although ISG15 was recently thought to function as an oncogene as well as a tumour-suppressor gene [10]. Enhanced ISGylation was observed in response to cancer chemotherapeutics, suggesting that ISG15 has a tumour-suppressing function. Moreover, ISG15 is a target of the tumour-suppressor gene TP53 [11]. In contrast, deregulated overexpression of ISG15 and enhanced ISGylation were positively correlated with cancerogenesis underlining the oncogenic potential of ISG15 [12-14]. As a secreted protein, ISG15 has been shown to primarily modulate immune cell activation [15].

Some of the ISG15 target proteins also have functions in the cell nucleus including chromatin remodelling and polymerase II transcription [16]. Interestingly, in bladder cancer immunohistochemistry revealed that ISG15 was located predominantly in the nuclei of cancer cells which were associated with an advanced tumour stage [12]. Surprisingly, ISG15 overexpression in human bladder cancer has not been found to be associated with a general inflammatory response even though ISG15 was expected to be stimulated by interferon primarily in an inflammatory process. Therefore, ISG15 expression in bladder cancer suggests that it is specifically associated with tumour cells [12].

In breast cancer, ISG 15 expression has so far only been examined in benign and malignant human breast cell lines [13]. To the best of our knowledge, this is the first study to systematically analyse the expression of ISG15 in human breast carcinomas and normal breast tissues both at the MRNA and the protein level. We analysed the results particularly in correlation to clinicopathological data, such as hormone receptor status, HER2 status and patient survival data, in order to validate ISG15 as a new prognostic marker and potential drug target in the treatment of human breast cancer.

\section{Materials and methods}

ISG15 protein expression was assessed in two independent cohorts of breast cancer patients using tissue microarrays (TMAs). The first tumour cohort has been previously described [17] and consisted of 179 breast cancer tissue specimens and 51 normal breast tissue specimens. The TMA used one tissue core from non-selected, formalin-fixed, paraffin-embedded primary breast cancer specimens from patients diagnosed between 1994 and 2002 at the Institute of Pathology, University of Regensburg, Germany. The patients' ages ranged from 25 to 82 years with a median age of 56 years. An experienced surgical pathologist $(\mathrm{AH})$ evaluated slides of all specimens, stained with haematoxylin and eosin (H\&E), before construction of the TMA in order to identify representative tumour areas. Histologically, all tumours were graded according to criteria by Elston and Ellis [18]. Clinical follow-up data, provided by the German Central Tumour Registry were available for all 179 breast cancer patients with a median follow-up period of 78 months (range 0 to 148 months). The Institutional Review Board of the participating centres approved the study.

The second TMA for validation consisted of 967 breast specimens, of which it was possible to analyse 646 breast carcinomas and 10 normal breast tissues samples. The second TMA was constructed from a cohort of breast cancer patients who were participants of a case-controlled trial for the assessment of breast cancer susceptibility markers and prognostic factors, the Bavarian Breast Cancer Cases and Controls (BBCC), which has been described previously, including methods for the data collection $[19,20]$. The database for this analysis closed on 31 December, 2007 and had a median follow up of 4.7 years. Paraffin embedded tissue was available for 967 BBCC patients. Briefly a $0.6 \mathrm{~mm}$ punch was retrieved from the breast cancer tumour after a pathologist (AD) evaluated H\&Estained slides of all specimens. Data about the hormone receptor status were obtained from original pathological reports of these specimens; the other data were collected from the patients' medical records.

The collection of paraffin-embedded and formalin-fixed normal ( $n=14)$ and cancerous $(n=25)$ breast tissue samples for the mRNA expression analysis has been described previously [21].

\section{Cell lines}

The human mammary epithelial cell lines HMEC and MCF12A, and the breast cancer cell lines MCF7, T47D, ZR75-1, MDAMB231, MDA-MB468, SKBR3 and BT20 were obtained from the ATCC (Rockville, MD, USA) and cultured as previously described [22].

\section{RNA extraction and reverse transcription}

Total RNA was isolated using the TRIzol reagent (Invitrogen, Carlsbad, CA, USA) according to the manufacturer's recommendations. For paraffin-embedded tissues, five consecutive 
sections (each $5 \mu \mathrm{m}$ thick) were prepared, deparaffinised and conventionally re-hydrated in a decreasing alcohol-series before RNA extraction. Of the obtained RNA, $1 \mu \mathrm{g}$ was reverse transcribed using the Reverse Transcription System (Promega, Madison, WI, USA). In order to improve the transcription rate oligo-dT was mixed with $\mathrm{pdN}_{6}$ primers at a ratio of $1: 2$.

\section{Semiquantitative real-time PCR}

Semiquantitative PCR was performed using the LightCycler system with the LightCycler DNA Master SYBR Green I Kit (Roche Diagnostics, Mannheim, Germany) as previously described [22]. To ensure the accuracy of the experiment, all reactions were performed in triplicate. Primer sequences used were:ISG15 sense 5'- GAG AGG CAG CGA ACT CAT CT 3' and antisense 5'- CTT CAG CTC TGA CAC CGA CA -3'; GAPDH sense 5'- GAA GGT GAA GGT CGG AGT CA -3' and antisense 5'- AAT GAA GGG CTC ATT GAT GG -3'. The annealing temperature for both genes was $60^{\circ} \mathrm{C}$. Reaction specificity was controlled by post-amplification melting curve analyses and gel electrophoresis of the obtained products.

\section{Breast cancer cDNA dot-blot hybridisation}

The breast cancer profiling array (BD Clontech, Heidelberg, Germany) contains 50 pairs of cDNAs generated from matched tumourous and normal breast tissue samples from individual patients and three breast cancer lymph node metastasis specimens, spotted on a nylon membrane [23]. Hybridisations using $25 \mathrm{ng}$ of a gene-specific ${ }^{32} \mathrm{P}$-labelled cDNA probe digested from Unigene cDNA clones were conducted according to the manufacturer's recommendations. The tumour/normal intensity ratio was calculated using a STORM860 phosphoimager (Molecular Dynamics, Sunnyvale, CA, USA) and normalised against the background.

Immunohistochemical studies for the expression of HER2 utilised an avidin-biotin peroxidase method with a 3,3'-diaminobenzidine chromatogen. After antigen retrieval (microwave oven for 30 minutes at 200W) immunohistochemistry studies were performed in a NEXES immunostainer (Ventana, Tucson, $A Z$, USA) according to the manufacturer's instructions. The primary antibodies used were anti-HER2 (DAKO, Hamburg, Germany; 1:400), anti-oestrogen receptor (ER) and anti-progesterone receptor (PR) (Novocastra, Newcastle Upon Tyne, UK; $1: 20)$. For target proteins, the ChemMate detection kit (DAKO, Hamburg, Germany) was used. A surgical pathologist (AH) performed a blinded evaluation of the TMA slides with no knowledge of clinical data. It was not possible to interpret some results for reasons that included a lack of tumour tissue and the presence of necrosis or crush artefacts. HER2 expression was scored according to the DAKO HercepTest. For the evaluation of the presence of ER and PR, a semiquantitative immunoreactivity score (IRS), as described by Remmele and Stegner [24], was used.

\section{ISG 15 Immunohistochemistry}

The TMAs of both the evaluation set and the validation set were subjected to immunostaining using the Advance Kit (K4068, DAKO, Hamburg, Germany) following the manufacturer's instructions. Paraffin-embedded breast carcinomas were used as positive controls. After deparaffinisation and rehydration the tissue samples were heated in a microwave oven for 30 minutes at $200 \mathrm{~W}$ in $10 \mathrm{mM}$ sodium citrate buffer (pH 7.2). Endogenous peroxidase was blocked by peroxidaseblocking solution (S2023, DAKO, Hamburg, Germany) for 10 minutes. The polyclonal primary antibody ISG15 (AP 1150a, rabbit, Abgent, San Diego, CA, USA) was applied (1:30 dilution) for 30 minutes at room temperature. The primary antibody was omitted in the negative controls. For signal detection 3,3'diaminobenzidine was used. Slides were counterstained with H\&E and after dehydration mounted in Vitro-Clud (Langenbrinck, Emmendingen, Germany). Two experienced pathologists (NB, AN) scored the immunohistochemical staining intensity according to the scoring system suggested by Remmele and Stegner [24].

\section{Statistical methods}

For statistical evaluation SPSS version 14.0 (SPSS Inc, Chicago, IL) was used. Differences were considered statistically significant when $p<0.05$. A non-parametrically two-tailed Mann-Whitney $U$-test was employed to analyse differences in expression levels. For analysis of the cancer profiling array, a Kolmogorov-Smirnov test was applied to test for a normal value distribution, followed by a two-sided paired $t$-test to analyse differences in normal and tumour expression. A statistical association between clinicopathological and molecular parameters was tested for using a two-sided Fisher's exact test. Recurrence-free survival (RFS) and overall survival (OS) were calculated according to the Kaplan-Meier equation.

For the BBCC-TMA, RFS was defined as any local recurrence or distant recurrence, whatever occurred first. Event-free survival (EFS) was defined as any local recurrence, distant recurrence or death, whatever occurred first. Kaplan-Meier estimates were used to display the survival curves and logrank test was used to compare patients with high vs. low ISG15 expression. For multivariate analyses, a Cox proportional hazard model was constructed for OS, EFS and RFS.

\section{Results}

Upregulation of ISG15 in human breast cancer cell lines

We started the study by investigating the level of ISG15 mRNA expression in non-malignant and malignant human mammary cell lines. Real-time PCR analysis revealed a low ISG15 mRNA expression among the non-malignant mammary epithelial cell lines HMEC and MCF12A, whereas ISG15 mRNA transcript levels varied more and in summary were clearly elevated in the cancerous cell lines BT20, MDAMB468, MDA-MB231, T47D and MCF7, of which MCF7 displayed an exceptionally high level of ISG15 mRNA (Figure 1). 
Figure 1

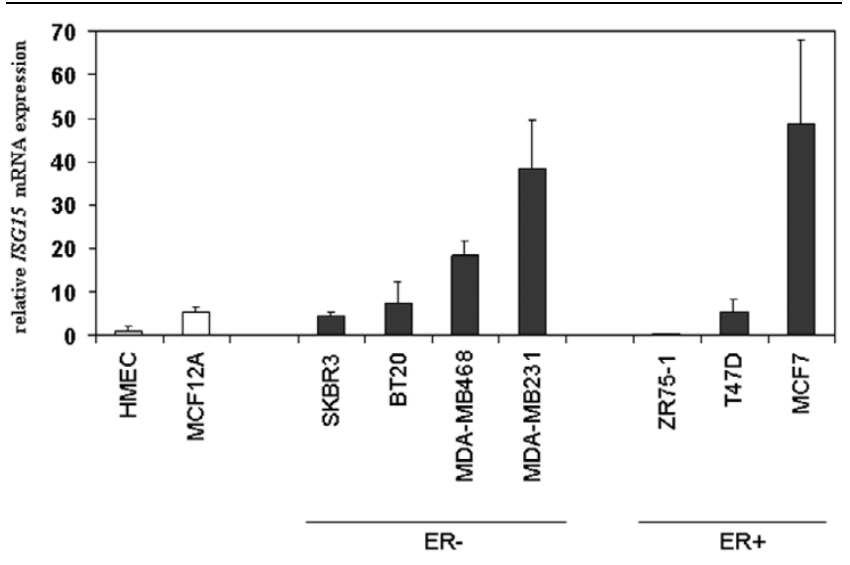

Abundance of the expression of ISG15 mRNA in breast cancer cell lines. Semiquantitative real-time PCR (LightCycler) of ISG15 expression was performed on reverse transcribed RNA from non-malignant (white bars) and malignant cell lines (black bars). ISG15 mRNA expression was low among the non-malignant mammary epithelial cell lines HMEC and MCF12A, whereas ISG15 mRNA transcript levels varied more and in summary were elevated in the investigated cancerous cell lines.

The median elevation of ISG15 expression in malignant cell lines vs. non-malignant cell lines was 2.5-fold and these differences in expression between the respective groups were statistically significant ( $p=0.043$, Mann-Whitney $U$-test). Differences in expression levels between ER-positive and ERnegative breast cancer cell lines were statistically not significant.

\section{Upregulation of ISG15 in primary breast cancers analysed by real-time PCR}

Next we analysed ISG15 mRNA expression in primary breast cancers $(n=25)$ and normal mammary samples $(n=14)$ derived from formalin-fixed, paraffin-embedded tissues by realtime PCR. In line with our breast cell line data, ISG15 mRNA was abundantly expressed in primary breast cancer specimens when compared with normal mammary epithelial tissue (Figure 2). The median fold change of ISG15 upregulation in the cancerous tissue vs. normal tissue was 10.3 and differences in expression between the two groups were statistically significant ( $p=0.002$, Mann-Whitney $U$-test).

\section{Correlation between ISG 15 mRNA expression and protein expression}

We additionally analysed 10 paraffin-embedded breast carcinomas and corresponding normal breast tissues by both semiquantitative real-time PCR and immunohistochemistry in order to correlate mRNA and protein levels in the same tumour samples as well as in the corresponding normal breast tissues. ISG15 mRNA was significantly upregulated in breast cancer specimens when compared with the corresponding normal breast tissues ( $p=0.003$, Mann-Whitney $U$-test). The median change in the tumour was three-fold compared with normal breast tissue on ISG15 mRNA level. At the protein level as determined by immunohistochemistry ISG15 was overexpressed in the tumour and correlated with upregulation of ISG15 mRNA. The median IRS in the tumour was five compared with an IRS of two in normal breast tissue and differences between the groups were statistically significant $(p<$ 0.01, Mann-Whitney U-test) (Figure 3).

\section{Upregulation of ISG15 in primary breast cancer analysed by cDNA dot-blot hybridisation}

ISG15 upregulation was validated by dot-blot analysis on a nylon array containing spotted cDNAs derived from 50 matched pairs of normal and cancerous breast tissue (Figure

Figure 2

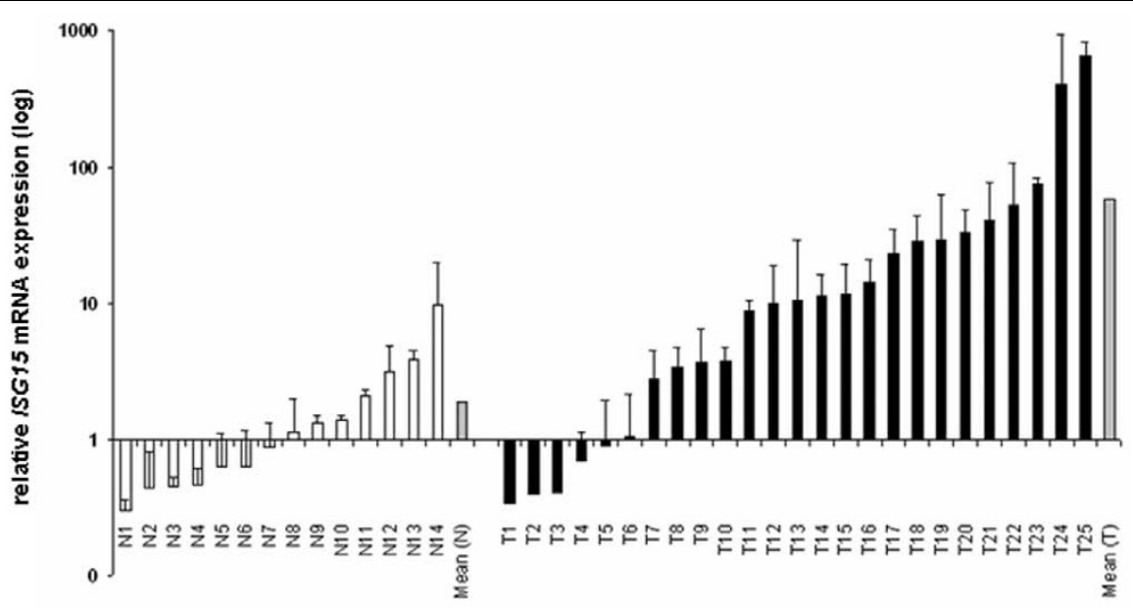

Upregulation of ISG15 mRNA in primary breast cancer. A collection of paraffin-embedded breast carcinomas $(\mathrm{T} ; n=25)$ and normal breast tissues $(\mathrm{N} ; n=14)$ was analysed for ISG15 expression by semiquantative real-time PCR. ISG15 was strongly upregulated at the transcript level in the tumourous tissues compared with the normal breast tissues. 

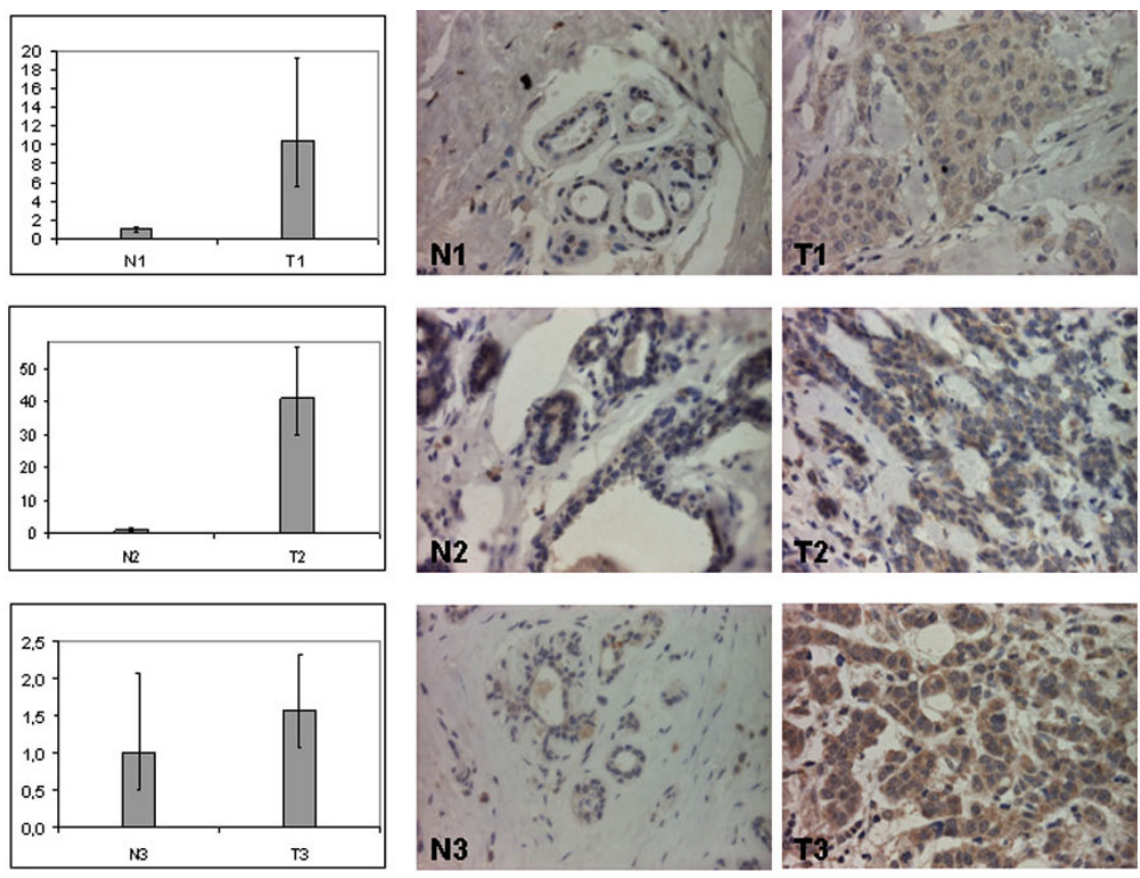

Correlation between ISG15 mRNA and protein expression. An additional collection of paraffin-embedded breast carcinomas $(\mathrm{T} ; n=10)$ and corresponding normal breast tissues $(\mathrm{N} ; n=10)$ was analysed for ISG15 expression by semiquantitative real-time PCR and immunohistochemistry in the same samples. ISG15 mRNA upregulation correlated with ISG15 protein expression in the same tumour sample (T) compared with the corresponding normal breast tissue (N). Magnification: 400x.

4). The cancer profiling array showed upregulation (fold change $\geq 2$ ) of ISG15 in 33 of 50 primary breast tumours $(66 \%)$, as well as in one of three metastatic lymph nodes, compared with matched normal breast tissue (two-sided paired $t$ test: $p<0.001$ ). Mean densitometric intensity in normal tissues was 1533 arbitrary units (standard deviation [SD] \pm 995 ) and 3571 arbitrary units (SD \pm 2292$)$ in tumour tissues.

\section{Overexpression of ISG15 protein in primary breast carcinomas}

Two independent cohorts of breast cancer specimens arranged on two different TMAs were analysed for ISG15 expression by immunohistochemistry. These represented an initial evaluation set, consisting of 179 breast carcinomas and 51 normal breast tissues, and an independent validation set, consisting of 646 breast carcinomas and 10 normal breast tissues. In normal breast tissue ISG15 expression was often absent or weak (Figure $5 \mathrm{a}, \mathrm{b}$ ). In ductal carcinoma in situ (Figure 5c, d) ISG15 expression was more intense than in normal breast tissue. In invasive breast carcinomas (Figure 5e, f, ductal type) ISG15 expression was generally more abundant than in either ductal carcinoma in situ or normal breast tissue. In tubular breast carcinomas (Figure $5 \mathrm{~g}, \mathrm{~h}$ ), a less frequent variant of invasive breast carcinomas with a more favourable prognosis than invasive ductal breast carcinomas, ISG15 expression was less abundant than in most invasive ductal breast carcinomas.
Statistical analysis of the initial evaluation TMA cohort Statistical data of the initial TMA cohort are presented in Additional files 1, 2, 3, 4. In the initial TMA cohort, ISG15 protein expression in breast carcinomas (IRS $>4$ ) was associated with the ER status $(p=0.028)$, but not with tumour size, lymph node status, histological grading, focality or histological type of tumour (Additional file 3). To investigate a possible impact of ISG15 overexpression on clinical outcome we calculated univariate survival probability curves with respect to immunohistochemical results. We found that ISG15 expression in breast cancer (IRS > 4) showed an unfavourable prognosis with regard to RFS as shown by Kaplan-Meier analysis ( $p=$ 0.012) (Additional file 1, Additional file 4). Patients who showed abundant ISG15 expression in the tumour had an estimated mean RFS of 112 months (95\% confidence interval [Cl]: 99 to 125) compared with 78 months (95\% Cl: 68 to 87) in patients with negative/weak ISG15 expression. Although OS was not significantly associated with ISG15 overexpression, there was a trend towards unfavourable prognosis in those patients with strong ISG15 expression (Additional file 2, Additional file 4). Possibly due to a weak association with the ER status, ISG15 expression could not be identified as an independent prognostic factor in a multivariate analysis in this breast cancer cohort. 


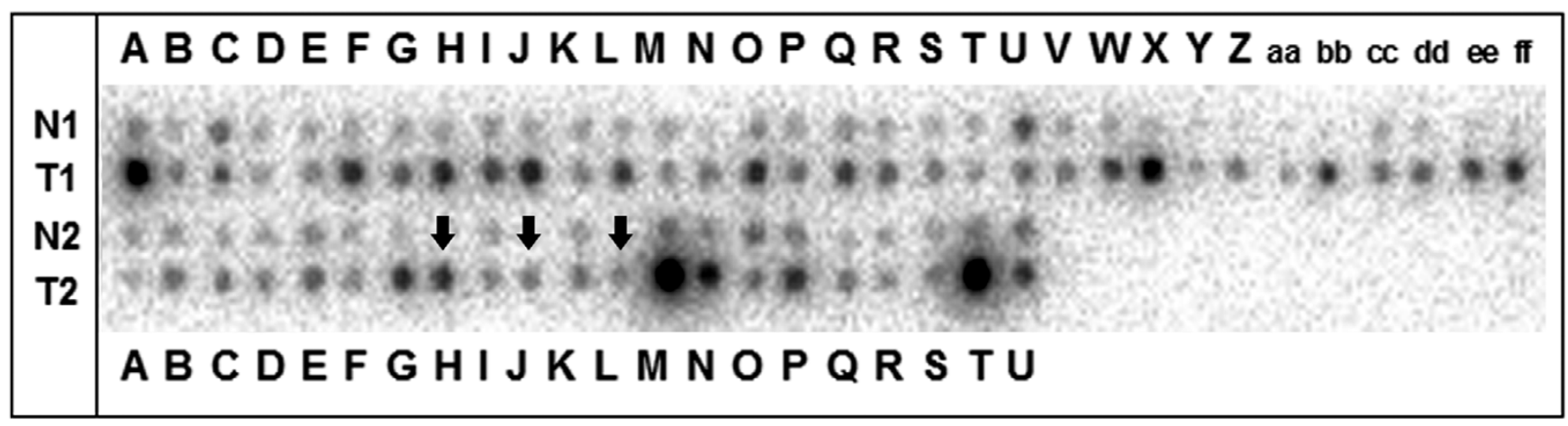

Expression profiles were determined using cDNA dot-blot hybridisation analysis (BD Clontech) containing cDNA pairs derived from 50 matched normal breast tissues $(\mathrm{N})$, tumourous breast tissues $(\mathrm{T})$ and three lymph node metastastic tissues (marked with arrows). Upregulation of ISG15 was observed in primary breast tumours and in one of three metastatic lymph nodes, as compared with matched normal breast tissue.

\section{Validation in the Bavarian breast cancer cases and controls tissue microarray cohort}

To confirm our results from the initial evaluation set of tumours, we analysed a second larger cohort (from BBCC) of breast cancer specimens on a TMA. In this validation cohort the IRS score was available for 646 invasive breast cancer cases. The mean IRS score was 3.5 with $83.9 \%(n=542)$ of tumours having a low score of zero to four and $16.1 \%(n=104)$ showing abundant expression of ISG15 (IRS > 4). As in the initial set we found a close association between ISG15 expression and an unfavourable prognosis (that is hazard ratio (HR) = $1.94 ; 95 \% \mathrm{Cl}: 1.17$ to $3.23 ; p=0.01$ in OS analysis). However, this cohort of tumours was divided into two groups of similar size for efficient correlation analysis. Therefore, the validation set of the additional 646 breast tumours was divided into low ISG15 expressers (IRS $=0$ to $3 ; n=333$ ) and high ISG15 expressers (IRS $=4$ to $12 ; n=313$ ). Under this slightly different cut-off level (IRS $>3$ ), the prognostic value of ISG15 expression was improved, as ISG15 immunohistochemical expression was also an independent predictor for OS and EFS in multivariate analysis (Table 1 and Figures 6 and 7). Furthermore, under this cut-off level, clear associations could be seen between ISG15 expression and both tumour size and grading (Table 2).

\section{Discussion}

ISG15 is a type I interferon regulated gene that is induced as a primary response to diverse microbial and cell stress stimuli [10]. Although the biological activities of ISG15 have yet to be elucidated, it is thought that ISG15 plays an important role in host defence and stress response pathways that have antitumour functions [10]. Consistent with this view, alterations in the ISG15 pathway have been identified in human tumours and in tumour cell lines. Increased expression of free and conjugated ISG15 has been exhibited in human breast, ovarian, prostate, colorectal and melanoma tumour cell lines compared with normal cell lines [13]. In accordance with the work of Desai and colleagues [13], who analysed two cell lines and showed abundant expression of ISG15 in the breast cancer cell line ZR-75-1 but not in the BT474 cell line, we also found upregulation of ISG15 mRNA in several additional breast cancer cell lines analysed (that is, BT20, MDA-MB468, MDA-MB231, T47D and MCF7) when compared with the benign mammary cell lines HMEC and MCF12A. Interestingly, upregulation of ISG15 was highly abundant in the breast cancer cell line MDA-MB231, a cell line shown to be highly metastatic in nude mouse models [25]. This could implicate a possible association between ISG15 upregulation and enhanced proliferation or invasiveness of cancer cells.

In agreement with the findings of ISG15 upregulation in diverse tumour cell lines in culture, microarray analyses of human tumour biopsies have revealed enhanced expression of ISG15 in pancreatic adenocarcinoma [14], endometrial cancer [13] and bladder cancer [12], when compared with the respective normal tissues. Expression of ISG15 in human cancerous and normal breast tissue has not been previously analysed. In the current study, a systematic characterisation of ISG15 expression in human breast cancer cells is presented for the first time at both the mRNA and the protein level, and includes a large cohort of breast carcinoma specimens that have been analysed by correlative analysis using clinicopathological parameters and patient survival data.

In accordance with previous findings in human cancer tissue [12-14] we found upregulation of ISG15 at the mRNA level in breast carcinomas compared with normal breast tissue. Enhanced expression of ISG15 in breast cancer was further validated by cDNA dot-blot analysis. Overexpression of ISG15 protein in breast carcinomas could be confirmed by immunohistochemistry of a TMA. Using this technique, ISG15 protein expression was correlated with patient survival data. In the initial cohort of breast carcinomas $(n=179)$ we found a significant correlation between ISG15 expression and unfavourable prognosis (RFS; $p=0.012$ ) suggesting a potential role of ISG15 in breast cancer development. Analysis of the second, 
Figure 5
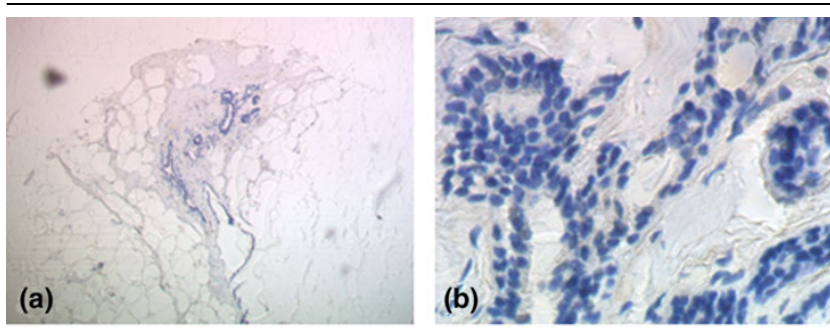

(c)
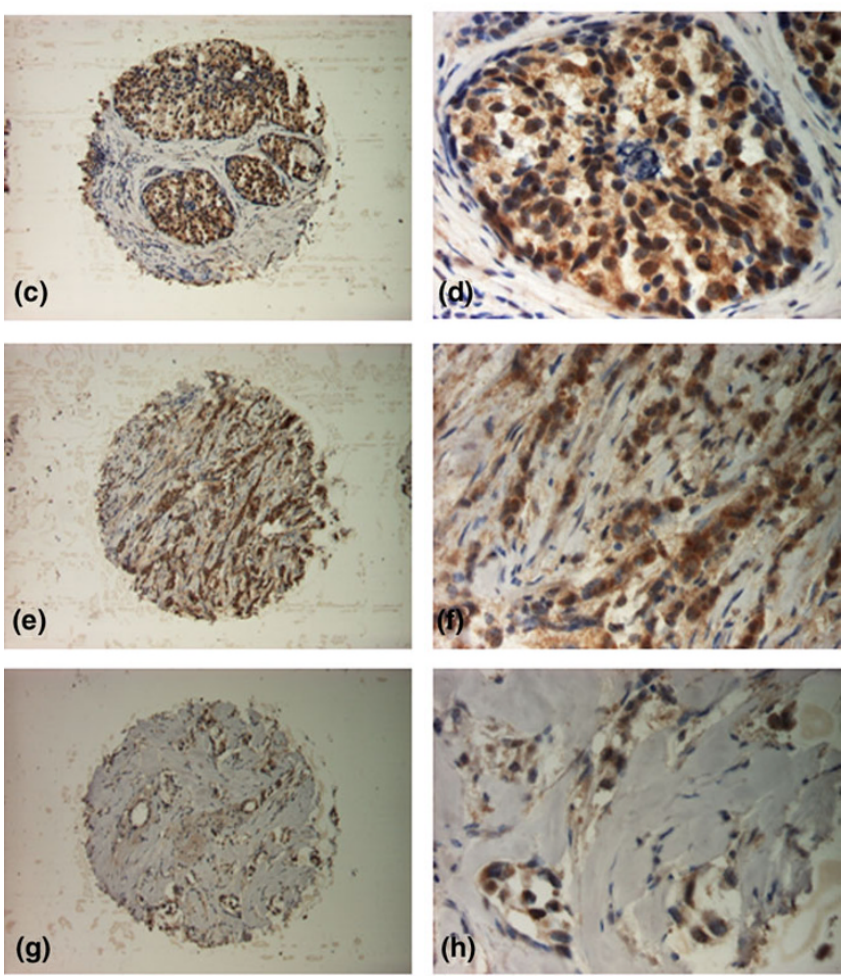

Immunohistochemical expression of ISG15 in normal breast tissue, and in non-invasive and invasive breast tumours using a tissue microarray. $(\mathrm{a}, \mathrm{b})$ Normal breast tissue lacks ISG15 expression (IRS =0). (c, d) In ductal carcinoma in situ ISG15 expression is more intense (IRS = 8) than in normal breast tissue. (e, f) In invasive breast carcinomas (here: ductal type) ISG15 expression was clearly more abundant (example shows staining with an IRS $=12$ ) compared with normal breast tissue and ductal carcinoma in situ. (g, h) In tubular breast carcinomas (IRS = 4), a less frequent variant of invasive breast carcinomas with a more favourable prognosis than invasive ductal breast carcinomas, ISG15 expression was less abundant than in most invasive ductal breast carcinomas and stronger than in most normal breast tissues. Magnifications: a, c, e, g: 100x; b, d, f, h: 400x.

independent breast cancer cohort $(n=967)$ confirmed this. IOn the validation TMA set, patients with ISG15 overexpression (IRS $>3$ ) showed unfavourable prognosis in RFS ( $p=$ $0.03)$, EFS $(p=0.001)$ and OS analyses $(p=0.001)$. This unfavourable prognosis of patients with abundant ISG15 expression might be due to an insufficient response to chemotherapy in this subgroup of patients. Indeed, deregulated ISG15 expression has been found in response to diverse cancer chemotherapeutic agents, such as paclitaxel in the treatment of ovarian carcinomas and 5-fluorouracil in the therapy of oesophageal cancer $[26,27]$. Bani and colleagues [26] analysed mice with human ovarian carcinoma xenografts undergoing treatment with paclitaxel as a way of understanding the in vivo molecular consequences of drug treatment. By analysing expression changes by cDNA microarray they found that paclitaxel could affect the level of expression of a variety of genes in those ovarian tumours responding to treatment. So the expression of several interferon-inducible genes such as ISG15 was reduced in the responders. Surprisingly, in human oesophageal squamous cancer cell lines, ISG15 was found to be upregulated after treatment with the chemotherapeutic agent 5-fluorouracil as shown by DNA microarray analysis and reverse transcription-PCR [27]. ISG15 mRNA expression was also found to be upregulated after treatment of human colorectal and breast cancer cell lines with campothecin, a topoisomerase I inhibitor $[28,29]$. Therefore ISG15 may be regulated differentially in response to different chemotherapeutic regimens.

Interestingly, ISG15 expression was associated with ER status in the initial TMA set $(p=0.028$; Additional file 3$)$, but not in the validation TMA set $(p=0.177$, Table 2$)$, as oestrogen is known to play a modulatory role in the ISGylation pathway. Indeed the ligating (E3) enzyme, that is a part of the post-translational modification by ISG15, was shown to be oestrogenresponsive [30]. The so-called ISGylation is mediated by a multi-step pathway, comprised of activating (E1), conjugating (E2) and ligating (E3) enzyme components [30]. The oestrogen responsiveness of the ligating (E3) enzyme in the ISGylation pathway is of great importance because ERs play a major role in the regulation of growth, survival and differentiation of normal and malignant breast epithelial cells $[31,32]$. Therefore, the determination of breast tumour hormone receptor status is of major importance for therapy selection [33]. Approximately 60 to $80 \%$ of all breast cancers abundantly express $E R \alpha$, but only two-thirds of those patients are responsive to endocrinal treatment (anti-oestrogen therapy). Intriguingly, a proportion of ER $\alpha$-positive tumours do not respond to hormone treatment at all (de novo resistance) while the majority of those tumours that initially responded to anti-oestrogens eventually become resistant during treatment (acquired resistance). Most ER-resistant tumours remain ER $\alpha$-positive, suggesting a continued role for ER $\alpha$ in breast cancer cell survival and proliferation $[34,35]$. The likely development of ER-resistance during breast cancer treatment with anti-oestrogens emphasises the urgent need for surrogate target molecules that may be able to bypass these resistances. Therefore, ISG15 might be a target molecule in the therapy of drugresistant breast tumours.

\section{Conclusion}

Our analyses showed ISG15 overexpression in human breast carcinomas relative to normal breast tissue at the RNA and protein level indicating that ISG15 may represent a candidate oncogene in human breast cancer. Interestingly, ISG15 could 
Table 1

Cox proportional hazard ratio analysis of the validation set (variables were categorised as indicated in Table 2)

\begin{tabular}{|c|c|c|c|c|c|c|c|c|}
\hline & & $n$ & $\begin{array}{l}\text { Overall Survival } \\
\text { Adjusted hazards } \\
\text { ratio }(95 \% \mathrm{Cl})\end{array}$ & $p$-value & $\begin{array}{l}\text { Event-free survival } \\
\text { Adjusted hazards } \\
\text { ratio }(95 \% \mathrm{Cl})\end{array}$ & $p$-value & $\begin{array}{l}\text { Recurrence-free } \\
\text { survival Adjusted } \\
\text { hazards ratio } \\
(95 \% \mathrm{Cl})\end{array}$ & $p$-value \\
\hline \multirow[t]{4}{*}{ Age } & $<50$ & 153 & 1 & & 1 & & 1 & \\
\hline & $50-59$ & 154 & $\begin{array}{c}1.19(0.54 \text { to } \\
2.63)\end{array}$ & 0.659 & $\begin{array}{c}1.15(0.67 \text { to } \\
1.96)\end{array}$ & 0.622 & $\begin{array}{c}1.20(0.69 \text { to } \\
2.09)\end{array}$ & 0.516 \\
\hline & $60-69$ & 158 & $\begin{array}{c}1.45 \text { (0.71 to } \\
2.97)\end{array}$ & 0.309 & $\begin{array}{c}0.99(0.59 \text { to } \\
1.67)\end{array}$ & 0.978 & $\begin{array}{c}0.81(0.46 \text { to } \\
1.43)\end{array}$ & 0.475 \\
\hline & $\geq 70$ & 106 & $\begin{array}{c}3.43(1.76 \text { to } \\
6.69)\end{array}$ & 0.0003 & $\begin{array}{c}1.94(1.17 \text { to } \\
3.22)\end{array}$ & 0.010 & $\begin{array}{c}1.17 \text { (0.64 to } \\
2.14)\end{array}$ & 0.612 \\
\hline \multirow[t]{2}{*}{$\mathrm{pT}^{\mathrm{a}}$} & $\mathrm{T} 1$ & 297 & 1 & & 1 & & 1 & \\
\hline & $\mathrm{T} 2-4$ & 274 & $\begin{array}{c}3.56(1.89 \text { to } \\
6.67)\end{array}$ & 0.00008 & $\begin{array}{c}2.88 \text { (1.85 to } \\
4.50)\end{array}$ & 0.000003 & $\begin{array}{c}3.258(1.95 \text { to } \\
5.43)\end{array}$ & 0.000006 \\
\hline \multirow[t]{2}{*}{$\mathrm{pN}^{\mathrm{a}}$} & 0 & 335 & 1 & & 1 & & 1 & \\
\hline & 1 & 236 & $\begin{array}{c}2.81 \text { (1.64 to } \\
4.82)\end{array}$ & 0.0002 & $\begin{array}{c}2.149(1.43 \text { to } \\
3.23)\end{array}$ & 0.0002 & $\begin{array}{c}2.52(1.57 \text { to } \\
4.04)\end{array}$ & 0.0001 \\
\hline \multirow[t]{2}{*}{ Grading } & 1 or 2 & 385 & 1 & & 1 & & 1 & \\
\hline & 3 & 186 & $\begin{array}{c}1.39(0.82 \text { to } \\
2.36)\end{array}$ & 0.218 & $\begin{array}{c}1.05(0.69 \text { to } \\
1.59)\end{array}$ & 0.832 & $\begin{array}{c}1.17(0.73 \text { to } \\
1.88)\end{array}$ & 0.513 \\
\hline \multirow{2}{*}{$\begin{array}{l}\text { Hormone } \\
\text { receptor status }\end{array}$} & Negative & 123 & 1 & & 1 & & 1 & \\
\hline & Positive & 448 & $\begin{array}{c}0.55(0.32 \text { to } \\
0.95)\end{array}$ & 0.032 & $\begin{array}{c}0.726(0.47 \text { to } \\
1.13)\end{array}$ & 0.160 & $\begin{array}{c}0.77(0.46 \text { to } \\
1.26)\end{array}$ & 0.287 \\
\hline \multirow[t]{2}{*}{ ISG15 } & Low (IRS 0 to 3 ) & 277 & 1 & & 1 & & 1 & \\
\hline & High (IRS 4 to 12 ) & 294 & $\begin{array}{c}1.78(1.07 \text { to } \\
2.95)\end{array}$ & 0.025 & $\begin{array}{c}1.516(1.03 \text { to } \\
2.22)\end{array}$ & 0.033 & $\begin{array}{c}1.39(0.90 \text { to } \\
2.13)\end{array}$ & 0.135 \\
\hline
\end{tabular}

aAccording to Sobin LH, Wittekind CH: UICC: TNM Classification of Malignant Tumours. $6^{\text {th }}$ edition, New York: Wiley; 2002. [36]. Cl, Confidence interval

function as a novel breast tumour marker with prognostic or predictive significance. Recently, microarray experiments determined strong deregulation of ISG15 expression in response to diverse cancer chemotherapeutic agents, for example, paclitaxel in the treatment of ovarian carcinomas or 5fluorouracil in the therapy of oesophageal cancer. These findings indicate that ISG15 could represent a predictive biomarker in the treatment of these cancers as well. However, the putative function of ISG15 in breast carcinogenesis and in chemotherapeutic response has to be further analysed in prospective studies. As a next step we will investigate whether an ISG15-based diagnostic assay is able to predict response to specific chemotherapy regimens in the treatment of breast cancer.

\section{Competing interests}

The authors declare that they have no competing interests.

\section{Authors' contributions}

NB: participated in the design of the study, data analysis, data interpretation, establishment and evaluation of the immunohistochemistry and drafted the manuscript; EN: carried out the immunohistochemical studies, and critically revised the manuscript; JV: supported with expertise in molecular biology techniques and in data interpretation and critically revised the manuscript; MFP: participated in construction of the BBCCTMA and data analysis; GK: supported in data interpretation and critically revised the manuscript; AN: participated in the evaluation and interpretation of the immunohistochemical data; $\mathrm{AH}$ : supported in data interpretation and critically revised 
Table 2

\begin{tabular}{|c|c|c|c|c|c|}
\hline Characteristic & & Low ISG15 (Score 0 to 3 ) & High ISG15 (Score 4 to 12) & Total $n(\%)$ & $p$-value \\
\hline Total & & $333(51.5)$ & $313(48.5)$ & $646(100)$ & $\mathrm{n} / \mathrm{a}$ \\
\hline \multirow[t]{2}{*}{ Age ( $t$-test) } & Mean $( \pm \mathrm{SD})$ & $57.3( \pm 12.2)$ & $59.2( \pm 12.4)$ & $58.2( \pm 12.3)$ & 0.053 \\
\hline & Total & 333 & 313 & 646 & \\
\hline \multirow[t]{5}{*}{ Age } & $<50$ & $91(52.3)$ & $83(47.7)$ & $174(100)$ & 0.05 \\
\hline & $50-59$ & $108(59.3)$ & $74(40.7)$ & $182(100)$ & \\
\hline & $60-69$ & $80(45.7)$ & $95(54.3)$ & $175(100)$ & \\
\hline & $\geq 70$ & $54(47.0)$ & $61(53.0)$ & $115(100)$ & \\
\hline & total & $333(51.5)$ & $313(48.5)$ & $646(100)$ & \\
\hline \multirow[t]{3}{*}{$\mathbf{p T}^{\mathrm{a}}$} & pT1 & $185(56.9)$ & $140(43.1)$ & $325(100)$ & 0.006 \\
\hline & PT2 to 4 & $139(45.9)$ & $164(54.1)$ & $303(100)$ & \\
\hline & Total & $324(51.6)$ & $304(48.4)$ & $628(100)$ & \\
\hline \multirow[t]{3}{*}{$\mathrm{pN}^{\mathrm{a}}$} & pNO & $193(52.9)$ & $172(47.1)$ & $365(100)$ & 0.504 \\
\hline & pN1 & $134(50.2)$ & $133(49.8)$ & $267(100)$ & \\
\hline & Total & $327(51.7)$ & $305(48.3)$ & $632(100)$ & \\
\hline \multirow[t]{3}{*}{ Grading } & 1 or 2 & $240(55.0)$ & $196(45.0)$ & $436(100)$ & 0.007 \\
\hline & 3 & $90(43.7)$ & $116(56.3)$ & $206(100)$ & \\
\hline & Total & $330(51.4)$ & $312(48.6)$ & $642(100)$ & \\
\hline \multirow[t]{3}{*}{ Hormone receptor Status } & Negative & $54(43.2)$ & $71(56.8)$ & $125(100)$ & 0.177 \\
\hline & Positive & $234(50.0)$ & $234(50.0)$ & $468(100)$ & \\
\hline & Total & $288(48.6)$ & $305(51.4)$ & $593(100)$ & \\
\hline
\end{tabular}

aAccording to Sobin LH, Wittekind CH: UICC: TNM Classification of Malignant Tumours. $6^{\text {th }}$ edition, New York: Wiley; 2002 [36]. SD, Standard deviation; n/a, not available; pT, Pathological assessment of the primary tumour; pN, Pathological assessment of the regional lymph nodes.

the manuscript; AD: did the histological review for all tumours included in the TMA and participated in TMA construction and data analyses, and critically revised the manuscript; MWB: supported in data interpretation and critically revised the manuscript; RK: participated in design and coordination of the study, and critically revised the manuscript; PAF: participated in construction of the BBCC-TMA, study design and coordination, data analysis, data interpretation and drafting of the manuscript; ED: conceived the study, participated in the study design and coordination, molecular and data analysis, data interpretation and drafting of the manuscript. 
Figure 6

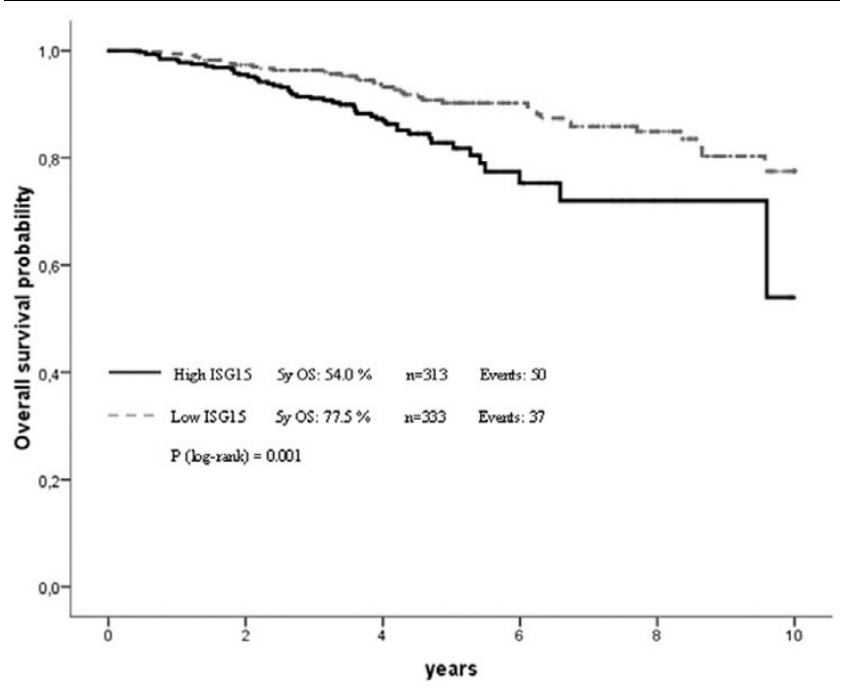

Correlation of ISG15 expression and patient prognosis according to univariate Kaplan-Meier analysis. Breast cancer patients overexpressing ISG15 show unfavourable prognosis in overall survival analysis ( $p=$ 0.001).

Figure 7

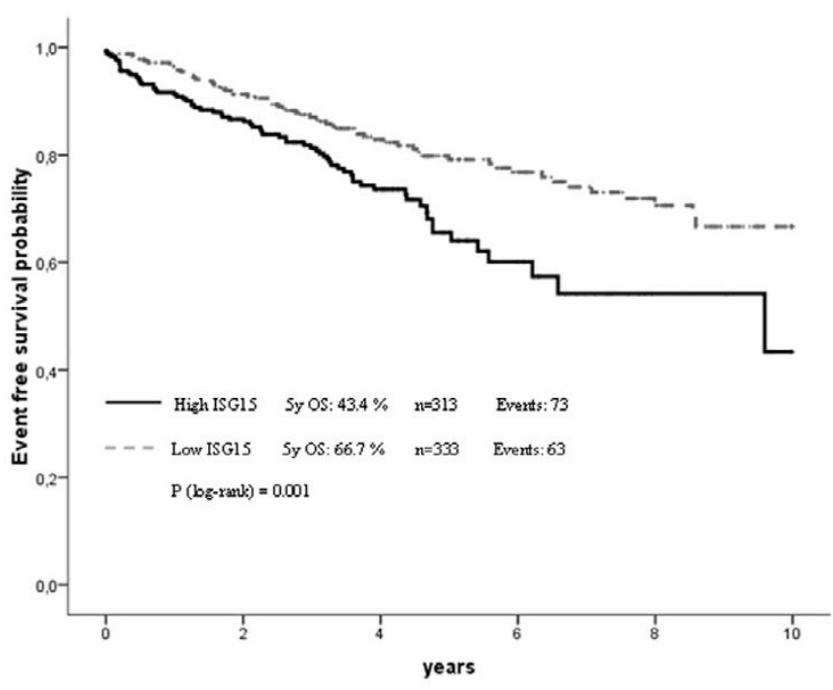

Correlation of ISG15 expression and patient prognosis according to univariate Kaplan-Meier analysis. Breast cancer patients overexpressing ISG15 exhibit unfavourable prognosis in event-free survival $(p=$ $0.001)$.
Figure 8

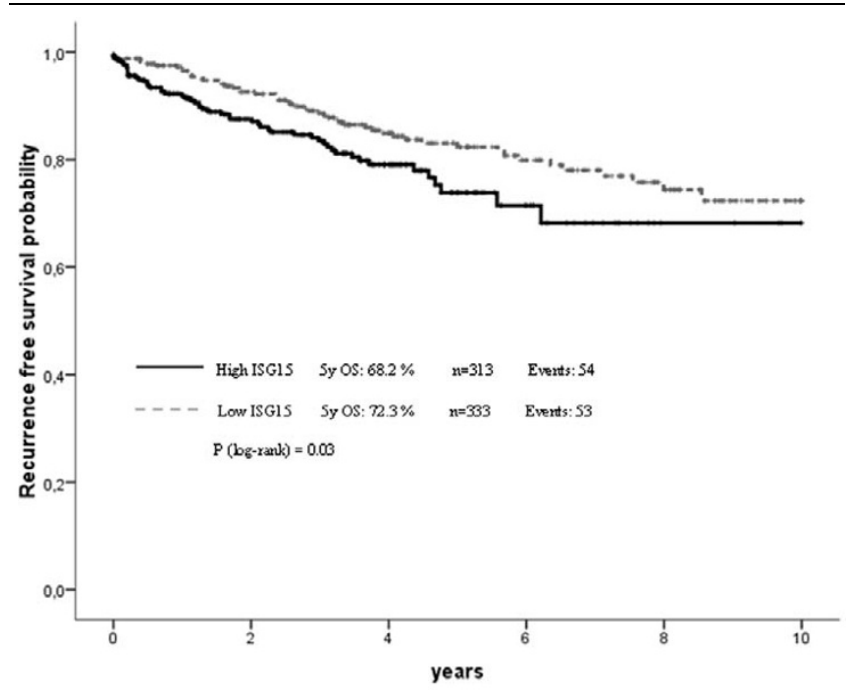

Correlation of ISG15 expression and patient prognosis according to univariate Kaplan-Meier analysis. Breast cancer patients overexpressing ISG15 have an unfavourable prognosis in recurrence-free survival analysis $(p=0.030)$.

\section{Additional files}

The following Additional files are available online:

\section{Additional file 1}

Tif file that shows the correlation of ISG15 expression and patient prognosis according to univariate KaplanMeier analysis in the initial TMA set. Breast cancer patients expressing ISG15 exhibit an unfavourable prognosis in recurrence-free survival analysis $(p=$ 0.012).

See http://www.biomedcentral.com/content/ supplementary/bcr2117-S1.tiff

\section{Additional file 2}

Tif file that shows correlation of ISG15 expression and patient prognosis according to univariate Kaplan-Meier analysis in the initial TMA set. Breast cancer patients expressing ISG15 show a trend towards unfavourable prognosis in overall survival analysis $(p=0.128)$.

See http://www.biomedcentral.com/content/ supplementary/bcr2117-S2.tiff

\section{Additional file 3}

Word file containing a table presenting the clinicopathological and immunohistochemical parameters in relation to ISG15 immunoreactivity in the initial TMA set.

See http://www.biomedcentral.com/content/ supplementary/bcr2117-S3.doc 


\section{Additional file 4}

Word file containing a table presenting the univariate analysis of factors regarding overall survival (OS) and recurrence-free survival (RFS) in the initial TMA set. See http://www.biomedcentral.com/content/ supplementary/bcr2117-S4.doc

\section{Acknowledgements}

The technical assistance of Inge Losen and Sonja von Serényi is greatly appreciated. The study was supported by the German Ministry for Education and Research as a part of the German Human Genome Project to PD Dr. Edgar Dahl and by a grant (START program) of the Faculty of Medicine, RWTH Aachen, to Dr. Nuran Bektas. We thank Ivonne Villalobos and Armen Gasparyan very much for their dedicated help constructing the BBCC-TMA.

\section{References}

1. Loeb KR, Haas AL: The interferon-inducible 15-kDa ubiquitin homolog conjugates to intracellular proteins. J Biol Chem 1992, 267:7806-7813.

2. Kim KI, Zhang DE: ISG15, not just another ubiquitin-like protein. Biochem Biophys Res Commun 2003, 307:431-434.

3. Der SD, Zhou A, Williams BR, Silverman RH: Identification of genes differentially regulated by interferon alpha, beta, or gamma using oligonucleotide arrays. Proc Natl Acad Sci USA 1998, 95:15623-15628.

4. Haas AL, Ahrens P, Bright PM, Ankel H: Interferon induces a 15kilodalton protein exhibiting marked homology to ubiquitin. $J$ Biol Chem 1987, 262:11315-11323.

5. Hamerman JA, Hayashi F, Schroeder LA, Gygi SP, Haas AL, Hampson L, Coughlin P, Aebersold R, Aderem A: Serpin 2a is induced in activated macrophages and conjugates to a ubiquitin homolog. $J$ Immunol 2002, 168:2415-2423.

6. Malakhov MP, Kim KI, Malakhova OA, Jacobs BS, Borden EC, Zhang DE: High-throughput immunoblotting. Ubiquitin-like protein ISG15 modifies key regulators of signal transduction. $J$ Biol Chem 2003, 278:16608-16613.

7. Giannakopoulos NV, Luo JK, Papov V, Zou W, Lenschow DJ, Jacobs BS, Borden EC, Li J, Virgin HW, Zhang DE: Proteomic identification of proteins conjugated to ISG15 in mouse and human cells. Biochem Biophys Res Commun 2005, 336:496-506.

8. Zhao C, Denison C, Huibregtse JM, Gygi S, Krug RM: Human ISG15 conjugation targets both IFN-induced and constitutively expressed proteins functioning in diverse cellular pathways. Proc Natl Acad Sci USA 2005, 102:10200-10205.

9. Liu YC, Penninger J, Karin M: Immunity by ubiquitylation: a reversible process of modification. Nat Rev Immunol 2005, 5:941-952.

10. Andersen JB, Hassel BA: The interferon regulated ubiquitin-like protein, ISG15, in tumorigenesis: friend or foe? Cytokine Growth Factor Rev 2006, 17:411-421.

11. Polyak K, Xia Y, Zweier JL, Kinzler KW, Vogelstein B: A model for p53-induced apoptosis. Nature 1997, 389:300-305.

12. Andersen JB, Aaboe M, Borden EC, Goloubeva OG, Hassel BA, Orntoft TF: Stage-associated overexpression of the ubiquitinlike protein, ISG15, in bladder cancer. Br J Cancer 2006, 94:1465-1471.

13. Desai SD, Haas AL, Wood LM, Tsai YC, Pestka S, Rubin EH, Saleem A, Nur-E-Kamal A, Liu LF: Elevated expression of ISG15 in tumor cells interferes with the ubiquitin/26S proteasome pathway. Cancer Res 2006, 66:921-928.

14. lacobuzio-Donahue CA, Maitra A, Olsen M, Lowe AW, van Heek NT, Rosty C, Walter K, Sato N, Parker A, Ashfaq R, Jaffee E, Ryu B, Jones J, Eshleman JR, Yeo CJ, Cameron JL, Kern SE, Hruban $\mathrm{RH}$, Brown PO, Goggins M: Exploration of global gene expres- sion patterns in pancreatic adenocarcinoma using cDNA microarrays. Am J Pathol 2003, 162:1151-1162.

15. Pitha-Rowe IF, Pitha PM: Viral defense, carcinogenesis and ISG15: novel roles for an old ISG. Cytokine Growth Factor Rev 2007, 18:409-417.

16. Jurica MS, Moore MJ: Pre-mRNA splicing: awash in a sea of proteins. Mol Cell 2003, 12:5-14.

17. Dahl E, Kristiansen G, Gottlob K, Klaman I, Ebner E, Hinzmann B, Hermann K, Pilarsky C, Durst M, Klinkhammer-Schalke M, Blaszyk H, Knuechel R, Hartmann A, Rosenthal A, Wild PJ: Molecular profiling of laser-microdissected matched tumor and normal breast tissue identifies karyopherin alpha2 as a potential novel prognostic marker in breast cancer. Clin Cancer Res 2006, 12:3950-3960.

18. Elston CW, Ellis IO: Pathological prognostic factors in breast cancer. The value of histological grade in breast cancer: experience from a large study with long-term follow-up. Histopathology 1991, 19:403-410.

19. Fasching PA, Loehberg CR, Strissel PL, Lux MP, Bani MR, Schrauder M, Geiler S, Ringleff K, Oeser S, Weihbrecht S, SchulzWendtland R, Hartmann A, Beckmann MW, Strick R: Single nucleotide polymorphisms of the aromatase gene (CYP19A1) HER2/neu status, and prognosis in breast cancer patients. Breast Cancer Res Treat 2007.

20. Schrauder M, Frank S, Strissel PL, Lux MP, Bani MR, Rauh C, Sieber CC, Heusinger K, Hartmann A, Schulz-Wendtland R, Strick R, Beckmann MW, Fasching PA: Single nucleotide polymorphism D1853N of the ATM gene may alter the risk for breast cancer. J Cancer Res Clin Oncol 2008, 134:873-882.

21. Zafrakas M, Chorovicer M, Klaman I, Kristiansen G, Wild PJ, Heindrichs $U$, Knüchel R, Dahl $E$ : Systematic characterisation of GABRP expression in sporadic breast cancer and normal breast tissue. Int J Cancer 2006, 118:1453-1459.

22. Veeck J, Niederacher D, An H, Klopocki E, Wiesmann F, Betz B, Galm O, Camara O, Durst M, Kristiansen G, Huszka C, Knuchel R, Dahl E: Aberrant methylation of the Wnt antagonist SFRP1 in breast cancer is associated with unfavourable prognosis. Oncogene 2006, 25:3479-3488.

23. Clontech/Information on the Cancer Profiling Array [http:// www.clontech.com/images/pt/PT3140-1.pdf]

24. Remmele W, Stegner HE: Recommendation for uniform definition of an immunoreactive score (IRS) for immunohistochemical estrogen receptor detection (ER-ICA) in breast cancer tissue. Pathologe 1987, 8:138-140.

25. Gallagher SM, Castorino JJ, Wang D, Philp NJ: Monocarboxylate transporter 4 regulates maturation and trafficking of CD147 to the plasma membrane in the metastatic breast cancer cell line MDA-MB-231. Cancer Res 2007, 67:4182-4189.

26. Bani MR, Nicoletti MI, Alkharouf NW, Ghilardi C, Petersen D, Erba E, Sausville EA, Liu ET, Giavazzi R: Gene expression correlating with response to paclitaxel in ovarian carcinoma xenografts. Mol Cancer Ther 2004, 3:111-121.

27. Matsumura $Y$, Yashiro M, Ohira M, Tabuchi H, Hirakawa K: 5Fluorouracil up-regulates interferon pathway gene expression in esophageal cancer cells. Anticancer Res 2005, 25:3271-3278.

28. Liu M, Hummer BT, Li X, Hassel BA: Camptothecin induces the ubiquitin-like protein, ISG15, and enhances ISG15 conjugation in response to interferon. J Interferon Cytokine Res 2004, 24:647-654.

29. Desai SD, Mao Y, Sun M, Li TK, Wu J, Liu LF: Ubiquitin, SUMO1, and UCRP in camptothecin sensitivity and resistance. Ann NY Acad Sci 2000, 922:306-308.

30. Horie-Inoue $\mathrm{K}$, Inoue S: Epigenetic and proteolytic inactivation of 14-3-3sigma in breast and prostate cancers. Semin Cancer $\mathrm{Biol}$ 2006, 16:235-239.

31. Khan SA, Rogers MA, Khurana KK, Meguid MM, Numann PJ: Estrogen receptor expression in benign breast epithelium and breast cancer risk. J Nat/ Cancer Inst 1998, 90:37-42.

32. Ricketts D, Turnbull L, Ryall G, Bakhshi R, Rawson NS, Gazet JC, Nolan C, Coombes RC: Estrogen and progesterone receptors in the normal female breast. Cancer Res 1991, 51:1817-1822.

33. Moy B, Goss PE: Estrogen receptor pathway: resistance to endocrine therapy and new therapeutic approaches. Clin Cancer Res 2006, 12:4790-4793.

34. Coombes RC, Powles TJ, Berger U, Wilson P, McClelland RA Gazet JC, Trott PA, Ford HT: Prediction of endocrine response 
Breast Cancer Research Vol 10 No 4 Bektas et al.

in breast cancer by immunocytochemical detection of oestrogen receptor in fine-needle aspirates. Lancet 1987, 2:701-703.

35. Taylor RE, Powles TJ, Humphreys J, Bettelheim R, Dowsett M, Casey AJ, Neville AM, Coombes RC: Effects of endocrine therapy on steroid-receptor content of breast cancer. $\mathrm{Br} J$ Cancer 1982, 45:80-85.

36. Sobin LH, Wittekind CH: UICC: TNM Classification of Malignant Tumours. 6th edition. New York: Wiley; 2002. 